\title{
Facebook Live como una nueva forma de consumir noticias. Una mirada al usuario digital peruano
}

\author{
Facebook Live as a new way to consume news. \\ An approach to the Peruvian digital user
}

Recibido: 31/08/2020

Aceptado: 09/11/2020

Publicado: 30/11/2020
Carolina L. Albornoz-Falcón

calbornozf@unmsm.edu.pe

https://orcid.org/0000-0001-7366-8210

Universidad Nacional Mayor de San Marcos (Perú)

Mariela Luján-Escribano marielalujanescribano@gmail.com https://orcid.org/0000-0003-0882-0065

Universidad Nacional Mayor de San Marcos (Perú)

Resumen: Desde su expansión en 2006, Facebook ha revolucionado no sólo la forma de comunicarse de la gente sino también de informarse. A través de Facebook Live (herramienta de reproducción de video en tiempo real) los usuarios pueden acceder a información multimedia y, a la vez, participar activamente en ella. Precisamente, esta plataforma es el objeto de estudio de la presente investigación. Mediante el análisis de contenido de las transmisiones en vivo de Facebook Live de los diarios La República y El Comercio, en su versiones digitales; y la emisora radial $R P P$, se analizó la forma cómo esta herramienta ha cambiado el modo de informarse de los usuarios digitales del Perú, cómo estos consumen las noticias a través de Facebook Live y cómo la convergencia mediática, la articulación hipertextual y la cultura participativa (características de los nuevos medios) están presentes también en las transmisiones en vivo que se analizaron para los fines de este estudio. 
Palabras clave: Facebook Live, Convergencia mediática, Articulación hipertextual, Cultura participativa, Usuario digital, Noticias.

Abstract: Since its expansion in 2006, Facebook has revolutionized not only how people communicate each other but also how they get informed. Through Facebook Live (a real-time video playback tool) users can access multimedia information and participate in it in the interactive way. This platform is the object of study of this research. Through the content analysis of the Facebook Live transmissions of daily newspaper La República and El Comercio, in their digital versions; and RPP news radio station, we discovered how this tool has changed the way the Peruvian digital users get informed; and also, how they consume the news through Facebook Live and how the characteristics of the new media as media convergence, articulating hypertext and participatory culture are present in the Facebook Live transmissions which were analyzed for the purposes of this study.

Key words: Facebook Live, Media convergence, Articulating hypertext, Participatory culture, Digital user, News.

\section{Introducción}

La actual revolución de la multimedia y el hipertexto, entendidos como la posibilidad de encontrar información desde múltiples hiperenlaces, ha puesto a la gente en un nuevo espacio donde la inmediatez, la interacción y la comunicación a distancia es posible con solo conectarse a una gran red que reúne todo: Internet.

Facebook forma parte de este nuevo espacio y ha ido imponiéndose en el mundo de las comunicaciones de manera progresiva desde su lanzamiento, hace 14 años (en el 2006). De acuerdo con We Are Social (2020), esta red social permanece en el primer puesto del ranking global de redes sociales con 2,449 millones de usuarios (data actualizada a enero de 2020), un 7,8\% más con respecto al año 2019. Entre las actividades más realizadas por estos usuarios activos se encuentra el compartir fotografías e información, participar en grupos y visualizar videos en directo o diferido a través de Facebook Live. La jefa de la aplicación de Facebook App, Fidji Simo (2017), informó 
en su cuenta personal de Facebook que 1 de cada 5 videos compartidos en la red social es un Facebook Live, la plataforma de transmisiones en vivo que es objeto del presente estudio.

El periodismo no es ajeno al uso de esta herramienta, en su propósito de estar en donde su público se encuentra, se ha amoldado a los cambios y actualmente ofrece cada vez más transmisiones en vivo como una nueva manera de informar. Esto forma parte de una revolución de la labor periodística que coincide con los nuevos gustos y necesidades de la audiencia. Al respecto, Trillo \& Alberich (2017) puntualizan que "la utilización de Periscope o Facebook Live para compartir información, la realización de infografías interactivas, la apuesta por los videojuegos, drones o la realidad virtual basados en información actual, son algunos de los ejemplos más populares que definen el nuevo ecosistema informativo" (p. 7).

Este nuevo ecosistema informativo ha reconsiderado también definiciones clásicas del perfil de los consumidores de los medios.

Si los viejos consumidores eran predecibles y permanecían donde les decías que se quedasen, los nuevos consumidores son migratorios y muestran una lealtad hacia las cadenas, las redes y los medios. Si los viejos consumidores eran individuos aislados, los nuevos consumidores están más conectados socialmente. Si el trabajo de los consumidores mediáticos fue silencioso e invisible, los nuevos consumidores son hoy ruidosos y públicos. (Jenkins, 2006, p. 29)

El público de hoy ya no es un espectador pasivo, sino un usuario activo que exige inmediatez, interacción y hasta participación. De este modo, quienes antes se informaban por medios clásicos (como la televisión, el periódico o la radio) ahora pueden hacerlo a través de una transmisión en vivo desde su computadora, tablet o teléfono móvil.

Este tipo de cambio (según la "teoría determinista" de las tecnologías de la información y la comunicación) se origina por el uso de una tecnología específica. "Para los teóricos de la Escuela de Toronto la comunicación está en el centro de las transformaciones sociales, y a cada nueva tecnología le corresponde una nueva forma de comunicación, un nuevo contenido y un nuevo uso" (Alberich \& Tubella, 2012, pp. 31-32). En esa línea, Facebook Live, 
que surgió en 2015 y que fue abierta a los usuarios en general en el 2016, ha impuesto una nueva forma de comunicación.

Actualmente, todos los medios importantes del mundo tienen una página de Facebook en donde ofrecen contenidos vía streaming. Los más usuales son entrevistas y coberturas de noticias; incluso se produce contenidos específicos para Facebook Live con formato televisivo, incorporando elementos propios de la televisión, tales como escenografía, mesa de conducción y horario regular de transmisión, sumando características propias de Facebook Live como es leer los comentarios de los usuarios en tiempo real (para contestar de manera personalizada) y hacer dinámica la transmisión. "Facebook Live es una herramienta perfecta para impulsar la interacción con la audiencia de un determinado programa durante su emisión (o no), o con el colectivo de "amigos" en esta red social, que reacciona ante los contenidos lanzados en directo" (Herrero, 2017, p. 527).

Pero, toda esta nueva cobertura a través de las redes sociales afecta también a los periodistas, quienes requieren más destrezas multimedias. "Es necesario volver a la idea del periodista global, al periodista con una formación integral ... La construcción de sus contenidos está fundamentada en las características distintas que posee Internet. El decodificador del mensaje no es un lector, un radioescucha o un televidente pasivo, es un protagonista de las tecnologías de la información y comunicación" (Navarro, 2009, p. 42). De este modo, el usuario digital se convierte en un protagonista activo, participativo y hasta crítico.

Así, el periodista tiene el desafío no solo de escribir bien, sino también de incorporar en su labor códigos audiovisuales, inmersivos y participativos. "Hay que abrirse a nuevos canales que exigen su propia arquitectura de escritura como Twitter, incorporar tecnologías como Periscope o Facebook Live que hacen real el principio de la inmediatez, y hasta bucear en nuevas experiencias narrativas y de producción con la incorporación de las tecnologías de realidad virtual y 3D del llamado periodismo de inmersión o con la utilización de drones" (Trillo \& Alberich, 2017, p. 4). Dicho sea de paso, los periodistas (de diferentes latitudes) ya se han apropiado de esa dinámica de trabajo.

En todo caso, ¿cómo Facebook Live está cambiando el modo de informarse de la gente?, ¿cómo los usuarios consumen las noticias a través de esta plata- 
forma?, ¿qué elementos de esta nueva herramienta se asocian al nuevo perfil del usuario digital peruano?

Para responder a estas preguntas de investigación, tomamos tres conceptos claves: "convergencia mediática", "cultura participativa" y "articulación hipertextual", que autores como Jenkins (2008), Alberich \& Tubella (2012), Castells (2001), Van Dijck (2016), Navarro (2009), Díaz-Noci \& Salaverría (2003), Caro \& Arbeláez (2009) y Manovich (2006) abordan en sus investigaciones para describir las características de los nuevos medios desarrollados a través de las nuevas tecnologías de información y comunicación. Tres términos que nos sirven para demostrar que estos principios también se presentan en los Facebook Live y explicarían cómo los usuarios digitales han cambiado el modo de informarse.

En cuanto al primer término (convergencia mediática) se entiende como el espacio "donde los antiguos y los nuevos medios chocan, donde las bases y los medios corporativos se entrecruzan, donde el poder de los productores de medios y el poder de los consumidores de medios interactúan de manera impredecible" (Jenkins, 2008, p. 14).

Para el autor la convergencia es el flujo de contenido a través de múltiples plataformas mediáticas donde confluyen los medios tradicionales o antiguos (prensa, radio y televisión) y los nuevos medios, la cooperación entre diversas industrias mediáticas y el comportamiento de las audiencias mediáticas que interactúan y se interrelacionan para encontrar su propia experiencia. Así, para Jenkins (2008), la convergencia representa un cambio cultural, "toda vez que se anima a los consumidores a buscar nueva información y establecer conexiones entre contenidos mediáticos dispersos" (p. 15).

En ese sentido, se trata de "profundos procesos de transformación que afectan al panorama de la comunicación en el nuevo siglo, un entorno donde los media no solo coexisten, sino que se interrelacionan y se influyen mutuamente en múltiples dimensiones, de acuerdo con un proceso [descrito por Jenkins] como Convergence Culture" (Alberich \& Tubella, 2012, p. 75). O lo que Castells (2001) denomina "caja mágica" o "multimedia" como un símil de la Internet que actúa como ancla fundamental para el sistema multimedia. Al respecto Castells (2001) señala que 
esta convergencia, desarrollada en términos un poco más analíticos ... en el que se mezclaban y se mezclan todos los mensajes, sea televisión, radio, periódicos, libros, cualquier medio de comunicación manejado, interactuado a través de Internet, e incluso abaratado es lo que yo he llamado hace tiempo la caja mágica, es decir, la caja multimedia, desde la cual se puede obtener todo lo que se quiere y acceder a todo mediante la fusión general de todos los medios de comunicación en un sistema informático interactivo. (p. 8)

De acuerdo con lo anterior, es común encontrar en la red y en Facebook Live, convergencia del conjunto de los medios de comunicación social; es decir, elementos característicos de la prensa tradicional, la televisión y la radio. Contenidos y noticias que reúnen texto, imagen y audio que están a disposición de los usuarios para que interactúen con ellos. Se trata de una cultura de convergencia en la que estamos inmersos todos: una convergencia tecnológica que tiene influencia en el modo cómo se producen las noticias y consumimos los medios, pues estamos acostumbrados a hacer varias cosas a la vez; a tener un papel dinámico y activo mientras nos informamos. El periodismo ha encontrado en Facebook Live la plataforma perfecta para cumplir esta expectativa del usuario digital.

El concepto de cultura participativa (el segundo término que analizamos en esta investigación) contrasta con nociones más antiguas del espectador mediático pasivo. "Más que hablar de productores y consumidores mediáticos, como si desempeñaran roles separados, podríamos verlos hoy como participantes que interaccionan conforme a un nuevo conjunto de reglas que ninguno de nosotros comprende del todo" (Jenkins, 2008, p. 18). De este modo, se impondría una característica clave del usuario digital que es el de participar en la producción de la noticia, a través de sus opiniones, críticas o aportes informativos como se ve en las transmisiones en vivo a través de Facebook Live.

Castells (2009) va un poco más allá y relaciona la cultura participativa con la "autocomunicación de masas" para explicar cómo la Internet ha enseñado al público a participar, opinar y aportar información:

Con la difusión de Internet, ha surgido una nueva forma de comunicación interactiva caracterizada por la capacidad para enviar mensajes 
de muchos a muchos, en tiempo real o en un momento concreto, y con la posibilidad de usar la comunicación punto a punto, estando el alcance de su difusión en función de las características de la práctica comunicativa perseguida. A esta nueva forma histórica de comunicación la llamo autocomunicación de masas. (p. 88)

De este modo, los lectores que antes eran receptores pasivos de la noticia, actualmente interactúan con ella, buscan experiencias y cooperan ofreciendo más información. "Ahora se busca atender a un usuario de la información que reclama más inmediatez, fiabilidad y una mayor participación en el proceso informativo" (Arrojo, 2015, p. 753). Es así como un contenido (que podría haber sido una noticia pequeña) puede convertirse en un Trending Topic online gracias a las colaboraciones del público. En este escenario, Facebook Live adopta un gran protagonismo, ya que el usuario interviene y abandona su anonimato para volverse un agente visible. Este usuario puede aportar nueva información durante el streaming e incluso asumir un rol protagónico, puesto que (dependiendo de su intervención o preguntas) podría marcar el sendero de la transmisión, así como direccionar la entrevista hacia los aspectos que a él le interese.

Otra forma de participación que tiene a su disposición el usuario es la de compartir la información, entendiendo este concepto como una de las acepciones que Van Dijck (2016) señala para conectar a la gente: "Impulsa a los usuarios a compartir información con otros a través de interfaces diseñadas para ello" (p. 51).

Del mismo modo, los emoticones "me gusta", "me encanta", "me asombra", "me entristece", "me enfada", también son una manera de participar en el streaming y es (al mismo tiempo) un feedback instantáneo para el medio, ya que le permite conocer las reacciones de la gente y corregir o enfatizar la información ofrecida. Toda esta cultura participativa ayuda también al periodista, quien con los aportes en simultáneo de los usuarios, podrá tener nuevas ideas y ángulos para abordar la información. Así, "la interactividad del periodista será otra fuente de información. Los usuarios comunican al medio informaciones y dudas, el periodista elaborará su trabajo de forma más completa. Estará en contacto directo con el usuario, podrá hablar con él, sabrá qué opina sobre su trabajo y qué información necesita" (Navarro, 2009, p. 42). 
Estos dos conceptos claves (la convergencia mediática y la cultura participativa) no podrían entenderse si no se analiza un tercer concepto: la articulación hipertextual, que propicia la interacción a través de múltiples hiperenlaces. Para hablar de articulación hipertextual o hipertextualidad tendríamos que definir primero este término, que tiene sus raíces en la palabra "memex", para referirse a una máquina conceptual de informaciones a la que el usuario podía acceder si tenía la habilidad de crear caminos, enlaces que llevasen de unas partes a otras como una forma de imitar el pensamiento humano asociativo. "Un memex es un aparato en el que una persona almacena todos sus libros, archivos y comunicaciones, y que está mecanizado de modo que puede consultarse con una gran velocidad y flexibilidad. En realidad, constituye un suplemento ampliado e íntimo de su memoria” (Bush, 1945, p. 14).

Esta acción fue denominada posteriormente por Ted Nelson como "hipertexto", a través del proyecto de 1960, "The Curse of Xanadu”, que iba más allá de una red compartida, buscando catalogar toda la información del mundo en un solo documento que almacene diversa información mediante enlaces hipertextuales (Wolf, 1995). En tanto, el prefijo "hiper" etimológicamente proviene del griego hyper, que significa "más allá de", "sobre", "encima de", "exageración". "Ted Nelson en los años 60 lo definió como ampliado, generalizado y multidimensional” (Díaz-Noci \& Salaverría, 2003, p. 86).

Por su parte, Caro \& Arbeláez (2009) también definen hipertexto como "un gran tejido de textos conectados que permiten pasar de un texto a otro" (p. 8). Entonces, podemos afirmar que el hipertexto hace referencia a mucha cantidad de textos que podrían estar conectados entre sí y que permite a los usuarios ampliar información, tener una red de contenido que le da contexto y ayuda a entender un tema. Según Caro \& Arbeláez (2009), la hipertextualidad es la propiedad de vincular textos en la red y está basada en el hipertexto, que a través de un sencillo código comunica una página con otra en Internet.

Del mismo modo, Manovich (2006) sostiene que "un hipervínculo crea una conexión entre dos elementos; por ejemplo, entre dos palabras en dos páginas diferentes, o entre una frase en una página y una imagen en otra, o entre dos distintos lugares dentro de la misma página. Los elementos que se conectan por medio de hipervínculos pueden existir en el mismo ordenador o en diferentes ordenadores conectados en red, como en el caso de la World Wide Web" (p. 15). 
Si trasladamos este concepto a la difusión de noticias vía Facebook Live, existe una articulación hipertextual que permite al usuario elegir qué recorrido tomar para buscar información, compartir el video en vivo en sus perfiles de Facebook o invitar a sus amigos en la red a seguir la transmisión. De esta manera, se hace uso de enlaces para seguir expandiendo la información y generar una estructura hipertextual para el resto de los usuarios.

Este nuevo ecosistema informativo descrito hace pertinente el presente trabajo de investigación, que busca conocer cómo Facebook Live está cambiando el modo de informarse de la gente, cómo los usuarios consumen las noticias a través de esta plataforma y qué elementos de esta nueva herramienta se asocian al nuevo perfil del usuario digital. Todo esto en el contexto peruano, a través de un análisis cualitativo de las transmisiones en vivo de Facebook Live de tres medios representativos como son las versiones digitales de los diarios El Comercio y La República; así como el sitio web de la emisora radial $R P P$.

El uso de Facebook Live (por parte de los medios de comunicación) es una tendencia creciente en el mundo, porque "los medios han visto también la oportunidad de captar audiencias y han comenzado a experimentar con herramientas como Facebook Live. El directo es la forma más viva de hacer televisión y, conjugar este formato con el potencial de las redes sociales, puede ser una vía muy eficiente para estimular la interacción con un gran número de seguidores" (Herrero, 2017, p. 530). Seguidores que crecen cada vez más, sobre todo, en el público joven.

Consideramos que estas innovaciones están sentando las bases de una nueva forma de consumir información, pero también de hacer periodismo, por lo que es esencial que los periodistas estén preparados para asumir el reto de ofrecer información con los mismos valores éticos que exige su profesión, ahora en un nuevo canal comunicativo digital como es Facebook.

Al ser un fenómeno relativamente nuevo en el Perú, es también un campo poco explorado, siendo este el motivo principal que nos impulsó a realizar la presente investigación que incluye los hábitos de consumo de noticias de los usuarios digitales en el país. Según Comscore (2019), la penetración de Internet en Perú, el acceso a dispositivos tecnológicos y las múltiples plataformas virtuales como redes sociales y la inmersión del contenido en streaming, han 
configurado a un nuevo consumidor digital en el país. En relación con el grupo etario, Comscore (2019) señala que la población online de Perú es joven en su mayoría. El 30,2 \% está conformado por personas de 15 a 24 años y el $21,1 \%$ oscila entre 24 y 35 años. En tanto, en un estudio de Ipsos Perú (2019), realizado en una población adulta de 36 a 59 años, se revela que el $72 \%$ es digital, el 70\% pertenece a alguna red social y el 50\% tiene un smartphone.

El conocimiento de este perfil digital del usuario peruano, sumado al análisis de contenido de diversos Facebook Live que realizamos para fines de esta investigación, nos permitió entender cómo interactúa el usuario digital peruano en la red y cómo Facebook Live le ha cambiado su manera de consumir noticias.

\section{Método}

En la presente investigación, empleamos una metodología esencialmente cualitativa que se identifica con la epistemología de la hermenéutica para comprender y describir las particularidades del cambio de hábitos en los usuarios que consumen noticias en nuevas plataformas digitales como Facebook Live. Con ello, nos asignamos la función de intérpretes o mediadores, tal como lo señalan Gordóvil \& Boixadós (2016) al postular que "el investigador que toma la concepción hermenéutica como referencia es concebido como intérprete, facilitador, mediador" (p. 8).

Como técnica de investigación, se ha empleado el análisis de contenido cualitativo de 12 transmisiones en vivo de noticias de Facebook Live, de la versión digital de tres medios representativos de Perú: emisora radial $R P P$ y los diarios El Comercio y La República, correspondientes al mes de diciembre del año 2019.

Los criterios considerados en la elección de las transmisiones noticiosas, lindan con la novedad y actualidad en sus contenidos y la inclusión de entrevistas a los protagonistas de la noticia. El tiempo de duración de las transmisiones oscila entre 5 a 30 minutos y se priorizaron temas informativos vinculados con la política, economía y locales (Tabla 1). 
Tabla 1

Muestra analizada para la investigación

\begin{tabular}{|c|c|c|c|}
\hline & $\begin{array}{c}\text { Diario El Comercio } \\
\text { (elcomercio.pe) }\end{array}$ & $\begin{array}{l}\text { Diario La República } \\
\text { (larepublica.pe) }\end{array}$ & $\begin{array}{c}\text { Emisora radial } R P P \\
\text { (rpp.pe) }\end{array}$ \\
\hline $\begin{array}{c}\text { TEMA } \\
1\end{array}$ & $\begin{array}{l}\text { Entrevista a Patricia An- } \\
\text { drade, viceministra del } \\
\text { Minedu, sobre los resul- } \\
\text { tados de la prueba PISA } \\
2018 . \\
\text { https://web.facebook. } \\
\text { com/elcomercio.pe/vi- } \\
\text { deos/498472887685107/ }\end{array}$ & $\begin{array}{l}\text { Elecciones 2020: Lesly } \\
\text { Shica vs. Lucía Alvites | } \\
\text { Versus Electoral. } \\
\text { https://web.facebook. } \\
\text { com/larepublicape/vi- } \\
\text { deos/719447645209363/ }\end{array}$ & $\begin{array}{l}\text { Costa Verde: ¿Por qué se dan los } \\
\text { desprendimientos de las rocas? } \\
\text { https://www.facebook. } \\
\text { com/rppnoticias/vi- } \\
\text { deos/766851110463223/ }\end{array}$ \\
\hline $\begin{array}{l}\text { TEMA } \\
2\end{array}$ & $\begin{array}{l}\text { ¿Quiénes reciben la } \\
\text { gratificación? ¿Cuál es } \\
\text { el plazo para el pago de } \\
\text { las gratificaciones de } \\
\text { diciembre? } \\
\text { https://web.facebook. } \\
\text { com/elcomercio.pe/vi- } \\
\text { deos/451372522246152/ }\end{array}$ & $\begin{array}{l}\text { Partidos ricos y famosos } \\
\text { - Tres D } \\
\text { https://web.facebook. } \\
\text { com/larepublicape/vi- } \\
\text { deos/1488994251254543/ }\end{array}$ & $\begin{array}{l}\text { Año Nuevo 2020: Control, } \\
\text { seguridad y precio de pasajes en } \\
\text { terminal terrestre Yerbateros. } \\
\text { https://web.facebook. } \\
\text { com/rppnoticias/vi- } \\
\underline{\text { deos/750183692143906/ }}\end{array}$ \\
\hline $\begin{array}{c}\text { TEMA } \\
\mathbf{3}\end{array}$ & $\begin{array}{l}\text { ¿Por qué la economía no } \\
\text { ha crecido como se tenía } \\
\text { previsto a inicios de año? } \\
\text { https://www.facebook. } \\
\text { com/elcomercio.pe/vi- } \\
\text { deos/561366117976735/ }\end{array}$ & $\begin{array}{l}\text { Essalud: Beneficios y } \\
\text { retos del sistema de salud } \\
\text { | RTV Economía. } \\
\text { https://web.facebook. } \\
\text { com/larepublicape/vi- } \\
\text { deos/2884844484881656/ }\end{array}$ & $\begin{array}{l}\text { Reconocido danzante de } \\
\text { Tijeras sufrió accidente y } \\
\text { denuncia negligencia en ope- } \\
\text { ración en el hospital Cayetano } \\
\text { Heredia } \\
\text { https://www.facebook. } \\
\underline{\text { com/rppnoticias/vi- }} \\
\underline{\text { deos/459996891549408/ }}\end{array}$ \\
\hline $\begin{array}{c}\text { TEMA } \\
\mathbf{4}\end{array}$ & $\begin{array}{l}\text { Entrevista a Graciela } \\
\text { Villasís, enviada espe- } \\
\text { cial de El Comercio a } \\
\text { Curitiba, Brasil. Nuevas } \\
\text { revelaciones sobre el Caso } \\
\text { Odebrecht. } \\
\text { https://www.facebook. } \\
\underline{\text { com/elcomercio.pe/vi- }} \\
\text { deos/2562188153818289/ }\end{array}$ & $\begin{array}{l}\text { Fiscalía acusaría a Keiko } \\
\text { Fujimori en febrero de } \\
2020 \text { | Sigrid.pe. } \\
\text { https://www.facebook. } \\
\text { com/larepublicape/vi- } \\
\text { deos/545015123015986/ }\end{array}$ & $\begin{array}{l}\text { Fiestas de Fin de año y Ve- } \\
\text { rano 2020: este es el plan de } \\
\text { Operaciones de Seguridad } \\
\text { Vial } \\
\text { https://www.facebook. } \\
\text { com/rppnoticias/vi- } \\
\underline{\text { deos/453438275567772/ }}\end{array}$ \\
\hline
\end{tabular}

Nota: Elaboración propia.

La etapa de acopio de datos, a través del análisis de contenido, como ya se mencionó líneas arriba, se llevó a cabo en el mes de diciembre del 2019, un mes preelectoral en Perú (por las elecciones extraordinarias legislativas que se realizó en enero 2020), donde la agenda de los medios estaba centrada en dicho acontecimiento, así también en la cobertura de noticias ligadas con la corrupción desatada por el escándalo continental de la empresa Odebrecht. 
Se realizó el análisis de contenido en torno a tres conceptos fundamentales de las nuevas herramientas digitales: la convergencia mediática, entendida como la fusión de los antiguos y nuevos medios; la cultura participativa, que convierte a los usuarios también en productores de noticias; y la articulación hipertextual, como la posibilidad de poder propiciar una interacción a través de múltiples hiperenlaces, presentes en las transmisiones noticiosas de Facebook Live.

Centrarnos en el análisis de esos tres elementos nos permitió aproximarnos a conocer cómo los usuarios peruanos están cambiando sus hábitos de consumo de noticias. Asimismo, nos llevó a identificar el patrón general de los contenidos y características de las transmisiones de noticias en la plataforma Facebook Live de los medios seleccionados.

\section{Resultados y discusión}

De acuerdo con el análisis de contenido realizado (sobre la base de las categorías analizadas: "convergencia mediática", "cultura participativa" y "articulación hipertextual") podemos exponer los siguientes resultados que nos ayudaron a comprender cómo Facebook Live está cambiando el modo de informarse de la gente, cómo los usuarios consumen las noticias a través de esta plataforma y qué elementos de los nuevos medios están presentes en las transmisiones en vivo de Facebook Live.

Con relación a la convergencia mediática, encontramos que los tres medios seleccionados para este estudio (los diarios El Comercio y La República; y la emisora radial $R P P$ ) integraron de diferente modo los elementos visuales televisivos, las características de prensa escrita y el lenguaje radial durante sus transmisiones en vivo a través de Facebook Live. Así, brindaron a los usuarios la posibilidad de informarse de manera audiovisual, interactiva y en tiempo real.

Dentro de los elementos televisivos, tales como el espacio o escenografía, El Comercio y RPP no emplearon ambientes tipo plató de televisión, como sí lo hizo el diario La República. Este último medio emplea una escenografía especialmente diseñada para sus transmisiones en vivo a través de Facebook Live, ofreciendo un ambiente televisivo que incluye una producción en maquillaje 
y vestimenta por parte del periodista. Por su parte, El Comercio realiza la transmisión vía Facebook Live en la misma sala de redacción periodística, donde se colocan unas sillas para el conductor y el entrevistado. En tanto, en la emisora radial $R P P$ se prioriza el empleo del escenario natural, es decir, transmisiones en exteriores (desde el mismo lugar de la noticia) que puede ser la calle, el interior de una casa o de una institución.

Si bien hay diferencias en los ambientes empleados para la transmisión en vivo entre estos tres medios de comunicación, todos usan un lenguaje televisivo en común, principalmente, en los diarios El Comercio y La República, donde el periodista emplea frases sencillas, repeticiones y buena vocalización. Con esto se logra contextualizar la información al usuario en diferentes momentos de la transmisión y, además, permite un mejor entendimiento del tema y fomenta la participación. Este aspecto también se observa en las transmisiones de $R P P$, pero en menor medida, ya que el periodista (en la muestra analizada) suele presentar el tema y al entrevistado al inicio de la transmisión, pero no lee los comentarios de los usuarios ni los invita a participar. Esto podría deberse a que las transmisiones en vivo son más cortas en $R P P$ (entre 6 y 9:53 minutos) y porque (como se mencionó anteriormente) se realizan en exteriores, por lo que el usuario tiene un reporte directo de la noticia, pero no mayor tiempo de análisis ni participación.

El uso de planos (característica de la narrativa televisiva audiovisual) también estuvo presente en la muestra analizada. En el Facebook Live del diario La República, se recurre al uso del plano general, medio y americano para contextualizar, fijar la atención en un personaje y mostrar a todos los integrantes de la transmisión, respectivamente. En este diario, incluso, se usa la doble pantalla o pantalla partida, con planos diferentes, en el que los usuarios pueden ver en una imagen a la periodista y en otra, al entrevistado.

En tanto, en el diario El Comercio se empleó el plano americano o medio, dependiendo del número de integrantes. Para los Facebook Live que incluyeron entrevistas, se empleó el plano americano durante toda la transmisión y el plano medio para resaltar la sola presencia de la periodista conductora. Por su parte, en $R P P$ se emplean planos americanos, medios y detalles, pero con la operación manual de un teléfono móvil. Realizar sus transmisiones en vivo con celular le permite a $R P P$ ofrecer un paneo de cámara para mostrar el lugar de la transmisión. Si bien esto aporta naturalidad y realismo a la toma, 
la calidad de la transmisión es inferior en comparación con El Comercio y La República. Así, los usuarios tienen la posibilidad de informarse de tres maneras, con una cobertura realizada desde una sala de redacción (diario $E l$ Comercio), un set de televisión (diario La República) o desde la calle (RPP).

En cuanto al empleo de los elementos de la prensa escrita, como parte de la convergencia mediática, se evidenció que el diario La República hace un mayor uso de estos recursos como la fotografía, infografía y elementos textuales. En todas sus transmisiones en vivo, se incluyeron cintillos (para recordar el tema abordado), textos relevantes, citas textuales o preguntas para propiciar la participación de los usuarios. Del mismo modo, el diario El Comercio empleó recursos escritos en el cintillo para dar a conocer el tema de la transmisión y el nombre del entrevistado, mientras que $R P P$ solo utilizó texto en el copy o resumen del post de Facebook, para explicar el tema.

Si de lenguaje radial se trata, encontramos que en algunos Facebook Live analizados se propicia la participación del público, tal como ocurre en la radio convencional. Así, se invita a los usuarios a dejar sus comentarios o preguntas. Sin embargo, esta práctica (que propicia la interacción) está perdiendo fuerza, pues sólo en el $25 \%$ de las transmisiones en vivo de El Comercio y La República se hace la invitación a los usuarios a participar. Esto podría deberse a que los Facebook Live no son realmente transmitidos en vivo, sino grabados con anterioridad y compartidos, como si se tratase de una retransmisión en vivo. Cabe acotar que, desde 2018, existe la funcionalidad denominada "Falso directo en vivo" o "Facebook video estreno" que la plataforma puso a disposición de los creadores audiovisuales.

En tanto, la emisora radial $R P P$ no suele hacer esta invitación al usuario. El periodista se enfoca únicamente en desarrollar la noticia, entrevistar a la fuente y cerrar la transmisión en vivo. La naturaleza de sus transmisiones vía Facebook Live (realizadas por lo general desde fuera de la emisora) y la corta duración de los mismos podrían ser los motivos por los cuales no existe esta interacción con los usuarios.

Así, en lo que corresponde a la primera categoría (convergencia mediática) podemos afirmar que la muestra analizada emplea recursos de medios tradicionales, tanto de la prensa escrita, radial o televisiva, para potenciar sus transmisiones en vivo vía Facebook Live y, de esta manera, captar la atención 
de los usuarios a través de un contenido informativo, dinámico y atractivo. Sin embargo, esta convergencia (de medios antiguos y nuevos) no se realiza en la misma intensidad en los tres medios analizados. En el diario $L a R e-$ pública hay más prevalencia del lenguaje televisivo y prensa escrita; en $\mathrm{El}$ Comercio y RPP predominan el lenguaje televisivo.

Por otro lado, la cultura participativa se presenta en la interacción de los usuarios, quienes tienen diversos niveles de participación a través de comentarios, reacciones mediante el uso de emoticones ("me gusta", "me encanta", "me enfada", me divierte, etc.), reproducciones de la transmisión en vivo o la posibilidad de compartir información.

Los usuarios de Facebook Live, tienen dos formas de informarse: en vivo y en diferido. En el primer caso, ellos interactúan con el periodista o entrevistado a través de comentarios o preguntas en tiempo real; en el segundo, no tienen la posibilidad de interactuar con el periodista o el entrevistado, pero sí con los demás usuarios que son notificados cuando deja un mensaje, ya que el video queda alojado en el Facebook del medio periodístico. La naturaleza de los textos (en este último caso) es para comentar u opinar lo que se abordó en la transmisión en vivo, así como para responder algunas interrogantes que quedaron sin contestar en el Facebook Live.

En los Facebook Live de larga duración, como los de los diarios El Comercio y La República (15 minutos y 24 minutos en promedio respectivamente), el número de comentarios y reacciones en tiempo real es mayor, mientras que en los Facebook Live de corta duración, como los de RPP (7 minutos en promedio), hay menos participación en tiempo real.

Es necesario resaltar que en ambos diarios (El Comercio y La República) primaron los comentarios y preguntas sobre lo que señalan los periodistas, pero también se pudo observar críticas y hasta cuestionamientos a ciertas afirmaciones vertidas en las transmisiones en vivo, generándose un debate entre los usuarios, quienes (en algunos casos) contaron sus propias experiencias. En otras, se limitan a responder la pregunta que el medio planteó al inicio del Facebook Live u ofrecen aportes informativos con datos o enlaces de páginas web con contenido complementario al tema, con lo cual se cumple con el concepto denominado "autocomunicación de masas", es decir, el usuario se convierte también en productor de la información. En tanto, en $R P P$, si bien 
hay comentarios, predominan las preguntas. Esto se debería a la corta duración de la transmisión en vivo, lo cual no permitió el debate. Sin embargo, sí hubo presencia de críticas hacia el periodista o el tema abordado.

De este modo, se produjo la interacción entre los mismos usuarios que, además, sirve de retroalimentación instantánea para el periodista. En los tres medios analizados se pudo observar intercambio de opiniones, etiquetas a otros usuarios que no están viendo la transmisión (como una forma de notificarles de la existencia de este video en vivo), reacciones a los comentarios de otros usuarios, a través de los ya conocidos emoticones.

Así, Facebook Live, permite que el usuario asuma un papel mucho más activo y participativo en el consumo de noticias, características que (antes de la llegada de esta herramienta digital) no existían, ya que, tradicionalmente, el consumo de información se realizaba de manera pasiva, sea leyendo un artículo, escuchando la radio o viendo la televisión. Este poder de interacción coincide con la afirmación de Fidji Simo en un artículo publicado en la página web oficial de Facebook (2016), donde señalaba que "Facebook Live es extremadamente interactivo. La experiencia muestra que las personas comentan 10 veces más durante la transmisión de un Live que en relación a videos comunes".

Respecto a la articulación hipertextual, característica de los nuevos medios que permite ampliar información e interactuar con otras fuentes, se encontró que El Comercio y RPP no añaden un enlace hipertextual para que los usuarios complementen información en algún artículo periodístico de su sitio web. No obstante, en todas las transmisiones en vivo del diario La República hubo presencia de enlaces que direccionan al usuario hacia la página web del mismo medio, donde este puede ingresar para expandir sus miradas sobre el tema puesto en agenda.

Con ello, podemos afirmar que, si bien la hipertextualidad es un aspecto importante del nuevo ecosistema digital, no todos los medios analizados lo emplean en beneficio de sus usuarios. En cambio, los usuarios sí lo consideran en sus comentarios o interacciones con la noticia, poniendo en evidencia lo que se denomina "cultura participativa". De esta manera, la articulación hipertextual puede ser aportada por el mismo medio que publica el URL de alguna nota periodística (relacionada con el tema de la transmisión en vivo) o por los 
mismos usuarios, quienes comparten diversos enlaces de noticias, videos o imágenes, iniciando así una cadena informativa.

En suma, los usuarios peruanos asumen un rol activo y crítico en el consumo de noticias. Hoy en día (a través de Facebook Live) aportan datos, intercambian opiniones y tienen el poder de elegir si se informan en tiempo real o en diferido, lo cual antes era imposible con el reinado de los medios tradicionales, que difundían sus contenidos noticiosos en un horario establecido. En definitiva, estamos ante usuarios que quieren sentirse parte de la noticia, conducir en alguna medida el rumbo de las informaciones y eso lo están encontrando en Facebook Live.

\section{Conclusiones}

Facebook Live representa una nueva era para los medios de comunicación, en un contexto donde la información e interacción es más inmediata y simultánea, y, a la vez, está cambiando los hábitos de consumo de noticias de los usuarios digitales peruanos, quienes interactúan con la información, opinan, critican y se convierten en productores de información al aportar nuevos datos. Esta plataforma permite que los usuarios de hoy puedan informarse de manera simultánea, cuando ocurren los hechos, y lo hacen a través de su teléfono móvil, desde el lugar donde se encuentren.

Las transmisiones en vivo de Facebook Live crean una audiencia que se identifican como una comunidad de usuarios y hasta pueden llegar a desarrollar un trabajo colaborativo o una interacción confrontacional. El primer caso, se evidencia a través de la comunicación que hay entre ellos al responderse algunas preguntas o aclarar unas dudas, mientras el segundo ocurre cuando (por puntos de vista opuestos) son capaces de agredirse verbalmente para defender su posición.

Así, a través de esta plataforma, se fomenta la autocomunicación y cultura participativa de los usuarios, pues les da poder y protagonismo en un escenario que antes les era ajeno. Ellos (cada vez más) quieren sentirse parte de la noticia y conducir (en alguna medida) el rumbo de las informaciones. Son más participativos, pues reaccionan a la noticia a través de múltiples vías: comentarios, reacciones mediante emoticones, enlaces para compartir información y etiquetas a otros usuarios para invitarlos a participar. 
Si hablamos de los elementos de los nuevos medios (la convergencia mediática, articulación hipertextual y cultura participativa) podemos afirmar que estos están presentes en las transmisiones en vivo de Facebook Live. Sin embargo, no a todos se les da la misma importancia. En cuanto a convergencia mediática, los tres medios analizados le dan mayor relevancia al lenguaje televisivo, en contraste con el lenguaje radial o de la prensa escrita. Por su parte, en la articulación hipertextual, solo el diario La República recurre a este elemento al colocar la URL de su página web. Sin embargo, los usuarios de los tres medios sí comparten enlaces para ampliar información. Mientras que la cultura participativa (fomentada por los medios) es limitada, pues no siempre se invita a los usuarios a dejar comentarios. En todo caso, estos sí participan voluntaria y activamente. En suma, se evidencia que existen diferentes estilos de Facebook Live, problema que podría ser abordado en una investigación ulterior.

Si bien los medios peruanos comprenden que su presencia en las redes sociales es crucial, están empleando cada vez menos la principal característica que ofrece Facebook Live, como es la simultaneidad de las transmisiones en vivo, que permite participar a los usuarios con sus preguntas o comentarios con la expectativa de recibir algún tipo de respuesta. Pero los medios, desafortunadamente, están optando por un "Falso directo en Facebook", hecho que está propiciando que no haya interacción real ni auténtica entre el periodista, el entrevistado y el usuario. Asimismo, el periodista no tiene posibilidad alguna de medir en vivo la aceptación o rechazo de su transmisión y, por tanto, no obtiene una retroalimentación instantánea.

En años recientes, el uso informativo de Facebook Live se ha convertido en una innovación periodística que los medios de comunicación peruanos han incorporado (cada vez más) a sus métodos de trabajo (casi de manera obligatoria), a fin de estar visibles para los usuarios digitales presentes en las redes sociales. Sin embargo, esto exige una mayor rigurosidad a los periodistas, quienes deberán asumir el desafío de ofrecer información desde múltiples plataformas digitales, sin que ese trabajo represente una disminución en la calidad informativa ni sea excusa para faltar a la ética profesional. Como tal, las empresas periodísticas también deberán valorar el esfuerzo del profesional de los medios. 


\section{Fuentes consultadas}

Alberich, J., \& Tubella, I. (2012). Comprender los media en la sociedad de la información. Editorial UOC.

Arrojo, M. (2015). Los contenidos transmedia y la renovación de formatos periodísticos: la creatividad en el diseño de nuevas propuestas informativas. Palabra Clave, 18(3), 746-787. http://www.scielo.org.co/ pdf/pacla/v18n3/v18n3a06.pdf

Bush, B. (2001). Cómo podríamos pensar. https://biblioweb.sindominio.net/ pensamiento/vbush-es.pdf

Caro, L., \& Arbeláez, N. (2009). Hipertextualidad, literacidad y discurso académico: conceptos para la gestión del conocimiento en la red. Revista Virtual Universidad Católica del Norte, 28, 1-23. https://www. redalyc.org/pdf/1942/194214468007.pdf

Castells, M. (2001). Un nuevo medio de comunicación: Internet. Treballs de comunicació, 17, 5-22. https://www.raco.cat/index.php/TreballsComunicacio/article/view/242866/325535

Castells, M. (2009). Comunicación y poder. Editorial Alianza.

Comscore. (2019). El consumidor peruano: digitalmente multiplataforma y cross-media. https://iabperu.com/wp-content/uploads/2019/10/ El-Consumidor-Peruano-Digitalmente-Multiplataforma.pdf

Díaz-Noci, J., \& Salaverría, R. (2003). Manual de redacción ciberperiodistica. Editorial Ariel.

Facebook. (2016,Abril 6). Facebook presenta nuevas formas de crear, compartir y descubrir videos Live. https://about.fb.com/ltam/news/2016/04/ facebook-presenta-nuevas-formas-de-crear-compartir-y-descubrir-videos-live/

Gordóvil, A., \& Boixadós, M. (2015). Las aproximaciones cualitativas y cuantitativas. Fundación para la Universitat Oberta de Catalunya. 
Goulart, M., Quesada, C., \& Furtado, R. (2019). Journalism social media: different profiles of journalistic content on the Facebook pages of Brazilian newspapers. Brazilian Journalism Research, 15(1), 176-199. https://doi.org/10.25200/BJR.v15n1.2019.1106

Guimarães, E. (2017). A cobertura jornalística das Olimpiadas 2016: apropriações do Facebook Live pelo SporTV [Tesis de maestría]. Repositorio institucional Universidade Federal da Paraíba. https://repositorio.ufpb.br/jspui/handle/tede/9584

Jenkins, H. (2008). La cultura de la convergencia de los medios de comunicación. Paidós.

Herrero, M. (2017). Nuevas fórmulas para la televisión en directo: el uso de Facebook Live en Atresmedia. Miguel Hernández Communication Journal, 8, 521-563. https://dialnet.unirioja.es/descarga/articulo/6120211.pdf

Manovich, L. (2006). El lenguaje de los nuevos medios de comunicación. Paidós.

Medeiros, K. (2018). Análise da cobertura jornalística em redes sociais digitais: o acontecimento rebelião em Alcaçuz veiculado nas lives do Facebook [Tesis de maestría]. Repositorio institucional de la Universidade Federal do Rio Grande do Norte. https://repositorio.ufrn.br/jspui/handle/123456789/25833

Navarro, L. (2009). Tres lustros del periodismo digital: interactividad e hipertextualidad. Comunicar, 17(33). 35-43. https://recyt.fecyt.es/index. php/comunicar/article/view/26330

Reuters Institute. (2018). Digital News Project 2018. https://reutersinstitute. politics.ox.ac.uk/sites/default/files/digital-news-report-2018.pdf

Simo, F. (2017, Abril 6). A year ago today, we made it possible for people around the world to share live video on Facebook [Post] Facebook. https://web.facebook.com/fidji.simo/videos/vb.576027062/10154258 $738027063 /$ 
Trillo-Domínguez, M., \& Alberich-Pascual, J. (2017). Deconstrucción de los géneros periodísticos y nuevos medios: de la pirámide invertida al cubo de Rubik. El profesional de la información, 26 (6). 1091-1099. https:// recyt.fecyt.es/index.php/EPI/article/view/epi.2017.nov.08/37048

Van Dijck, J. (2016). La cultura de la conectividad: Una historia crítica de las redes sociales. Siglo Veintiuno Editores.

We Are Social. (2020). Digital In 2020: Global Digital Overview. https:// wearesocial.com/digital-2020

Wolf, G. (1995, Enero 6). The curse of Xanadu. Wired. https://www.wired. com/1995/06/xanadu/ 